\title{
Review of: "Different theta connectivity patterns underlie pleasantness evoked by familiar and unfamiliar music"
}

Ying Liu

Potential competing interests: The author(s) declared that no potential competing interests exist.

The main idea of the study is clear and supported by proper data analysis.

However, when put forward the existing research lack of the connectivity dynamics between the brain nodes associated with pleasantness differentiated by familiar music, the theoretical significance and practical value of doing are not stated.In addition, there are 5 males and 17 females in the subject group.But the paper is lack of corresponding explanation and explanation for this unbalanced sex ratio, which is insufficient. Besides, the acronym ISPC is not marked with its full name when it first appears in the text, which should be added. Finally, the discussion is not thorough enough. The relationship between musical familiarity and pleasure is not fully explained. The EEG evidence here supports part of the hypothesis, and further data are needed to clarify the relationship between the familiarity and music pleasantness. 